\title{
Slide-Rule Remedy To Numbers Desensitized Students
}

\author{
Vlastimil Masek \\ Associate Professor, Faculty of Engineering and Applied Science \\ Memorial University of Newfoundland \\ masek@mun.ca \\ May 6, 2011
}

\begin{abstract}
The rapid technological advancement of past decades changed significantly the way young engineers are educated these days. A large selection of numerical tools widely available on personal computers or pocket calculators is being applied where tables, nomograms or a slide rule were used in the past. It has been observed that despite of the gained time efficiency, high versatility and extreme precision, some students became largely affected through overuse of these tools and as a consequence became unable to verify obtained results by common sense and estimation. This paper presents once orthodox, currently unorthodox approach of using a highly customized circular-slide-rule and an associated simulator in electrical engineering courses in order to assist students develop a feel for what a sensible answer ought to be and thus help students to become less likely to make errors in the order of magnitude or false precision. This is because questioning a result in slide rule calculations is a necessary step leading to decimal point errors being de facto eliminated. By using the slide rule and practicing mental math to determine the result's order of magnitude, students gradually become conscious of scales as well as become aware of precision and tol-
\end{abstract}

erance implications in engineering. Our slide rule can be customized in terms of a number of mathematical functions available plus the unused empty space can be populated by frequently used formulas or conversion tables specific to an individual subject area. Our slide rule was first applied in a classroom in 2007 with not so wide acceptance and reintroduced in 2011 with more positive results. A number of examples with classroom observations including a student feedback are presented.

\section{Introduction}

Proper understanding of engineering concepts can be judged by abilities of analyzing, designing and solving real-world physical problems. These are often practiced at school in lectures, labs, tutorials, or at home through written assignments or self-study. Nearly every engineering textbook has a large portion of the text devoted to problems and exercises. Also as in other professions including sports, practicing means maintaining or improving own skills. Benjamin Franklin's “Tell me and I may forget, show me and I may remember, involve me and I'll understand" is a classical example indicating the importance of getting involved in a process rather then just being told a result. Students involvement in solving engineering prob- 
lems thus constitutes an essential part of their learning.

When tools such as calculators are used in problem solving students often omit or neglect the importance of verifying the results whether intermediate or the final one. We can confidently say the calculators are trustful but this does not apply to the process of entering and reading the numbers or typing the functions or operators by us. Humans are error-prone but there is no spell checking equivalent available for these things. Once a digit or decimal point is mistyped or misread the result is wrong which could have disastrous consequences in the real world. Performing a dimensional and unit checking with result verification is therefore another essential part of good engineering education and profession.

In addition, with the current trend of moving towards more and more complex systems and devices, such as in the communications or automotive industry, it seems inevitable more and more complex problems are also entering engineering textbooks. Especially in the latest multidisciplinary approach to many problems, we nowadays often consider all the interactions and details at once while making fewer assumptions and simplifications. Multimedia with embedded computer simulations enabled conveying such complex engineering concepts in a classroom. We can now present large picture system interactions in action which helps to better understand the covered concepts. A large variety of the specialized programs and software is available to students to perform the complex analyzes and syntheses, however by doing so more often these days, students have gradually less practice in the number crunching of simpler problems where result validation is strait forward.

Less and less frequently we use estimates to verify the final numerical results provided by calculators or software, mainly because it is an option these days and not a necessity known from past. Reintroducing the result estimation and verification as a required part of problem solving may thus reinforce student confidence and may trigger additional thinking about the problem and its result consequences. This tends to significantly eliminate commonly seen answers such as a circuit resistor tolerance being determined as $\pm 30 \%$ or a thermocouple delivering $k V^{\prime} s$ of thermo-electric force.

The majority of student solved engineering problems do not also need to be executed super-fast as the computational time efficiency is often not critically needed. In fact the manual\&mental calculation may lead to faster results in some cases. Majority of engineering problems also rarely requires computations to be carried out with accuracies of more than three significant digits. Many times I witnessed a resistor in an electronic circuit being evaluated to five, six or even more significant digits. The high number of significant digits implies in high manufacturing accuracies that would lead in our resistor example to a need for laser trimming that is rarely required. An approximate evaluation can be justified in many situations also due many engineering systems include safety factors or a way to compensate for real-world imperfections, such as using fine trimming/setting components like potentiometers or set screws.

The above experience led to introducing a now obsolete slide rule (SR) back into the classroom. Many articles has been written about this tool after its extinction in late 70's or early 80's [1], [2], and many books on SR are still being published these days. However, many of them address the evolution and history of the SR and its many variants while others are mostly reprints of the older texts. Many sites in the cyber-space are also devoted to the SR calculations and some even include printable templates for a custom SR assembly. I found the templates either too complex with too many scales crammed into a small 
space, or too simple without special marks for mostly used constants such as the base of natural (Napier) logarithms $e$ or $\pi$. Therefore a printable circular slide rule that is highly customizable for any particular field has been developed in a response to the unsuccessful efforts in finding a contemporary work on using a SR in modern engineering classroom.

\section{Circular Slide Rule}

The Circular Slide Rule (CSR) was programmed using a vector graphics language Asymptote that provides a natural coordinatebased framework for technical drawing. Labels and equations are typeset with $\mathrm{HT}_{\mathrm{E}} \mathrm{X}$ for high-quality postscript output.

Originally the size of the large disk was designed to meet a regular CD/DVD disk diameter to enable using the LightScribe technology of printing CSR as a disk label, however, the contrast of the scales on the disk background was rather poor, quite shiny and such hard to read. It has been found that a regular 600dpi or higher resolution laser printer, black\&white or color, produces better quality scales that last sufficiently long under normal use.

Besides the ability of customizing the number and relative order of the SR scales on four discs, such as $A, B, C, D, K, C I, D I, S, T$, $S T, S R T, L, L L n$ among others, the empty space near the center of the smaller disc can also be utilized to list frequently used formulae or diagrams. Figure 1 shows an example of the inner disc with $C L$ scales customized for Electrical and Computer Engineering (ECE) students enrolled in the Electronic Circuits I.\&II. courses, while Figure 2 illustrates another inner disc with $L L_{2}$ and $L L_{3}$ scales designed for ECE students taking Industrial\&Process Control And Instrumentation courses where magnetic concepts in sensing and actuation are covered. Frequently used constants can be also be added to the scales.

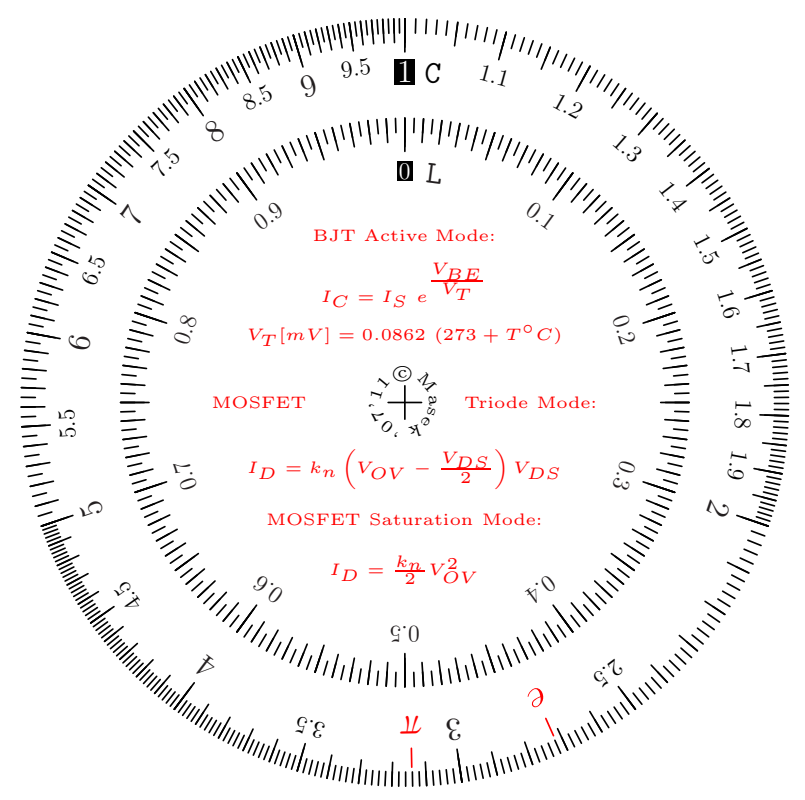

Figure 1: Inner disc with $C L$ scales.

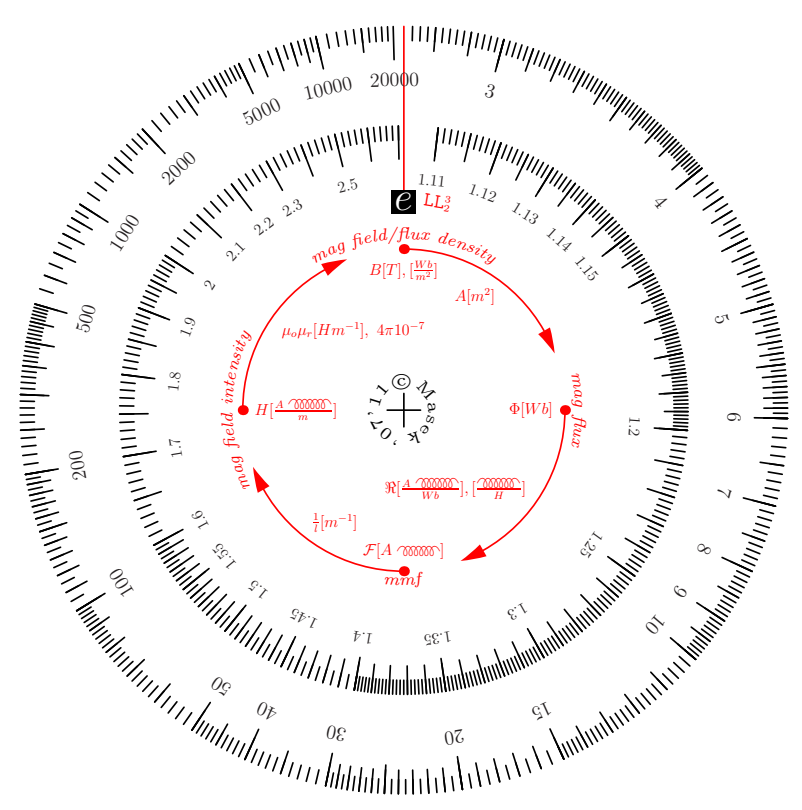

Figure 2: Inner disc with $L L$ scales. 


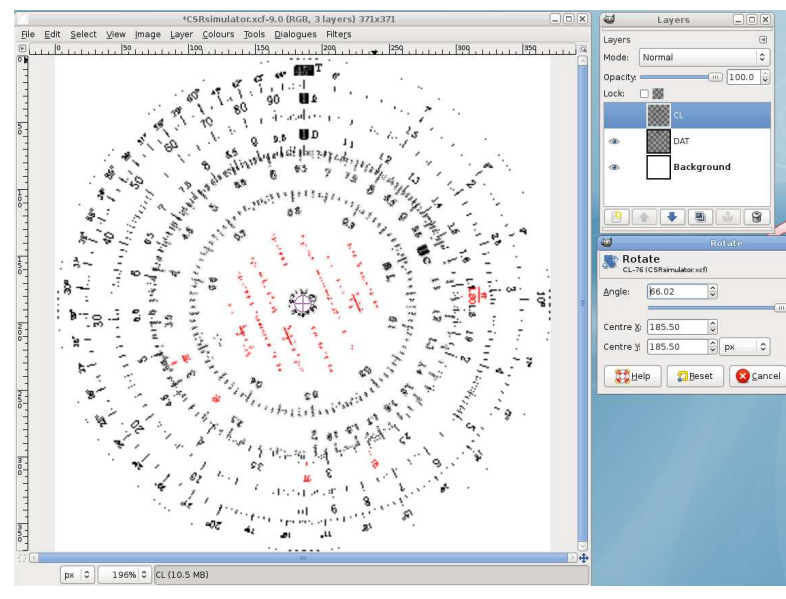

Figure 3: CSR simulator using GIMP

\section{Circular Slide Rule Simulator}

For teaching purposes, a custom built SR simulator is currently being developed. It will support such functions as flipping the CSR to get reciprocal values $1 / x$ instantaneously without adding CI or DI scales.

Meanwhile, a simple alternative simulator based on the GNU Image Manipulation Program (GIMP) is being used. In GIMP, individual discs are stored in separate layers positioned concentrically, where the inner (smaller) disc has transparent outer margins (corners) to allow the larger disc underneath being visible at any time (Figure 3).

Even simpler but equivalently efficient method uses the display camera now available in almost every Memorial's classroom. This way the students also see how to use fingers to move the scales quickly and precisely.

\section{CSR in Classroom, 2007}

The CSR was first introduced in Term 5 Control Systems I. course for ECE students in 2007. All students prepared and assembled own CSR by themselves in the second week of their classes using their unused lab slot. Despite of its simplicity, we needed to work precisely since small misalignments or eccentricities between individual discs would result in a
SR having poor accuracies.

First, students punctured centering pinholes of the two large discs (outer scales), stuck a pin into the holes and glued the two discs backto-back. It was important to accurately align the origins of both $D$ scales on each side in order to create a reciprocal scale identical to $D I$ scale. Students used few techniques for this such as pinning the discs down at the mark associated with the primary number 1 on the $D$ scale of both discs, called the index. Then they cut out the three discs, two small ones (inner scales) and the one large created in the previous step, along the circumference and punched larger holes for the center rivet. This step is the most critical for accuracy as the concentricity is one of the most important parameter for an accurate CSR. We used a punch tool with a spring loaded centering pin for this operation. The second last operation was to cut out and fold in half a transparent runner with a hairline imprinted on it, called the indicator. Finally, the two smaller discs on each side of now double-sided large disc were rivetted together with the indicator inserted last on top of the discs on both sides. It shall be mentioned that six plastic foil washers was added between the smaller and larger discs and also the indicator and the pivot to reduce the friction and extend the lifespan of this analog computing tool. Despite of its simplicity, it took a considerable effort to make own CSR and by doing so, students learned an important lesson on accuracy and precision.

The CSR usage was introduced in a tutorial session using a chalkboard as we did not have a CSR simulator at that time. We covered a range of operations including multiplication $(x \times y)$, division $(x / y)$, reciprocal values $(1 / x)$, square roots and squares $(\sqrt{x}), x^{2}$, decadic or natural logarithms $\left(\log _{10} x, \ln x\right)$, exponentiation $\left(10^{x}, e^{x}\right)$ and trigonometric functions $(\sin x, \arcsin x, \tan x, \arctan x)$ including conversion between radians and angular 
degrees. Both $S$ and $T$ scale for the trigonometric functions start from $6^{\circ}$, which students quickly pointed out as a major deficiency and a reason to drop the SR requirement. Fortunately the both functions can be approximated as $\sin x \approx x[\mathrm{rad}]$ and $\tan x \approx x[\mathrm{rad}]$ for small angles with a sufficient accuracy. In our particular case, the error of approximating the sine function for angles under $6^{\circ}$ results in errors under $0.2 \%$ at maximum which closely meets the maximum SR accuracy of reading the scales anyway (3 significant digits or $0.1 \%)$. Thus there is no real need for adding $S T$ scale for small angles on our CSR where space is limited. The conversion between radians and degrees can be done using $\pi / 180$ mark on both $D$ scales or even more conveniently by just reading it out on the $S$ scale.

A portion of the tutorial was devoted to determining the order of magnitude (decimal point location) through mental math and estimation. This is a necessary operation in SR calculation but redundant when calculators are used. This is however one of the main motivations behind the SR introduction which cultivates an intuition for numerical relationships and scale.

Students were also given web links for further study on the SR techniques since we did not spent multiple tutorials on practicing these techniques but continued covering the course subject. It was found too ambitious to assume a wide acceptance of SR among students that were so familiar to using their pocket calculators. As a result, in two weeks, the CSR idea all fell apart and the requirement of using the CSR was removed under the intervention of ECE Discipline Chair. After this negative experience, continuing in the idea became the farthest thing to consider because such feedback is unwanted by an untenured faculty. Not all responses were critical though as some called it highly regrettable the CSR requirement was dropped.

\subsection{CSR Reintroduction in 2011}

The SR idea was reintroduced in Winter 2011 Term 5 Electrical Circuits II. course after correcting Midterm Tests. It has been observed that one quarter of the class of fifty students had serious problems when calculating with exponential and logarithmic functions, and also problems in getting results with the correct order of magnitude which again originated mostly from difficulties in combining multiple exponents.

The following example from classroom illustrates a real situation where calculator obtained result was incorrect but would be widely accepted if not questioned by the instructor. A square wave generator using Schmitt trigger circuit was discussed and the class evaluated the frequency of oscillation for some particular component values as:

$$
f=\frac{10^{3}}{2 \ln 3}[H z]
$$

Students were asked to find the result and numerically evaluate the frequency. A quick answer from the first row desk was $4.55 \mathrm{~Hz}$ off by two decimal places! The rest of the class was quiet so without revealing the mistake, students were asked second time to verify the result. Fortunately his time the calculators gave correct result. Having knowledge of the base $e$ and the natural logarithm function $\ln ()$ one can draw a good estimate much faster than using a pocket calculator in this case. This is another example that has led to reintroduction of the SR concept.

This year, students had an option to purchase the SR for \$2 or build their own from an online posted template, or continue using calculator since the SR was not made compulsory. Fifty CSR's were made for each student but only a half of the class bought one. More time was spent on the SR practice this time but it should come earlier in the term to see more impact. 
Despite of the brief introduction and use, the data from Final Exam analysis show that those students without trying the SR had 50\% more problems with logarithms than those that had some exposure to SR calculations. Unfortunately, only two students out of fifty recognized a given voltage of 2.718 Volts as ' $e$, which consequently led to a very simple evaluation in one of the problems on the exam.

A small questionary was also conducted among those students that received the SR. Two thirds (17 students) responded effectively with the outcome plotted in Figure 4 but one third (8 students) returned $100 \%$ negative response with no comments. These highly biased students that did not spend time and showed lack of respect to providing the feedback were eliminated from the analysis. The seventeen students felt quite strong about seven out of eight questions/statements showing a general interest and acceptance of the SR. This is however at a condition, that this tool is continued at a voluntary basis and not made mandatory, especially at tests or exams (Q6). This gives some indication that students are unlikely to change their habits of using calculators quickly due the above mentioned benefits such as speed, accuracy and easiness.

In summary, the slide rule shall be implemented on longer time and wider usage basis to start seeing any significant difference. Despite of the brief nature of SR exposure this term, seeing few students using the slide rule at the Final Exam was the most positive feedback one could get at this time as it shows trust in the instrument and desire to be machine independent and somewhat creative in numerical calculations.

\section{Conclusion}

A new approach to assist students in becoming less dependent on calculators in solving less complex problems and less likely to make errors in the order of magnitude or false pre-

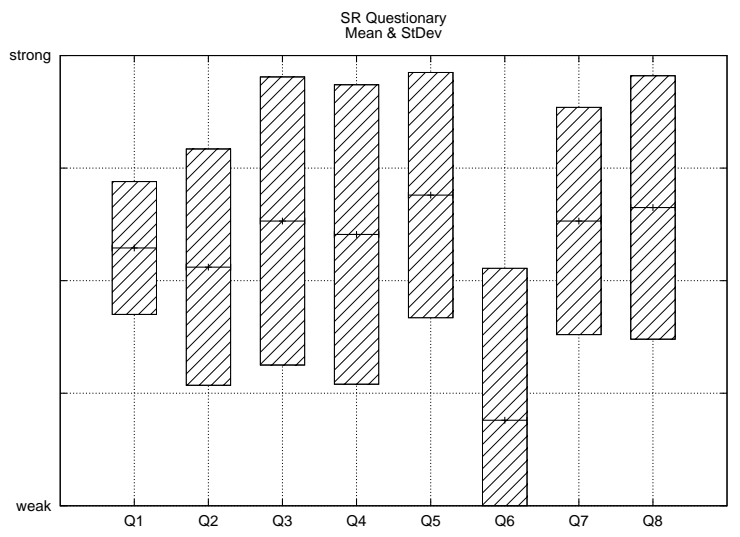

Q1: I found the SR useful

Q2: I will continue using my SR

Q3: I wish I had the SR earlier

Q4: I know better how logs work now

Q5: I do not mind deciding on decimal point using common sense

Q6: SR shall be mandatory for all students at tests and exams

Q7: I wish to know how to add other scales

Q8: I wish to customize my next SR

Figure 4: SR Questionary Results

cision has been introduced by complementing calculators by a customized slide rule. Initially, the technique has not met with a large acceptance among term five students, however, the latest reintroduction shows some positive signs. The plan is to continue in development of a new curriculum that targets using more mental math and estimation that will likely give our students more confidence in their professional life.

\section{References}

[1] Harvey Dearden, "Bring Back The Slide Rule?", IEE Engineering Management, August/September 2005, p.45

[2] Robert Kanigel, "Lament for the Slide Rule", IEEE Power Engineering Review, August 1985, p.12 